Check for updates

Cite this: RSC Adv., 2017, 7, 42064

Received 30th May 2017

Accepted 16th August 2017

DOI: $10.1039 / c 7 r a 06048 c$

rsc.li/rsc-advances

\section{Nitrogen-rich green leaves of papaya and Coccinia grandis as precursors of activated carbon and their electrochemical properties}

\author{
Paweena Dulyaseree, (D) ab Masatsugu Fujishige, ${ }^{\mathrm{b}}$ Ichiro Yoshida, ${ }^{\mathrm{b}}$ Yumiko Toya, ${ }^{\mathrm{b}}$ \\ Yasuo Banba, ${ }^{\text {b }}$ Yu-suke Tanaka, ${ }^{C}$ Takaaki Aoyama, ${ }^{c}$ Mayuree Phonyiem, ${ }^{a}$ \\ Winadda Wongwiriyapan, (D) ${ }^{* a}$ Kenji Takeuchi ${ }^{\star b}$ and Morinobu Endo ${ }^{b}$
}

Activated carbon (AC) was synthesized from papaya and Coccinia grandis leaves (PL-AC and CL-AC, respectively) which are nitrogen-rich precursors and their electrochemical properties were investigated. The synthesis process included carbonization at $400{ }^{\circ} \mathrm{C}$, impurity removal by $\mathrm{H}_{2} \mathrm{SO}_{4}$ cleaning, and post activation by $\mathrm{NaOH}$ at $720^{\circ} \mathrm{C}$. Surpassing the conventional bamboo-derived $A C$ (B-AC), PL- and CL-ACs show relatively high surface areas of 2664 and $2576 \mathrm{~m}^{2} \mathrm{~g}^{-1}$, respectively. Moreover, the nitrogen contents in the PL- and CL-ACs were approximately 2.3 and 1.8 at\%, respectively. Furthermore, the electrochemical properties of the synthesized PL- and CL-ACs were investigated using both aqueous and organic electrolytes. The specific capacitances of the PL- and CL-ACs were 98.47 and $89.91 \mathrm{~F} \mathrm{~g}^{-1}$, respectively, in $\mathrm{Na}_{2} \mathrm{SO}_{4}$ electrolyte. Especially, compared to the B-AC, the PL-AC shows a dramatic decrease in series resistances $\left(R_{\mathrm{S}}\right)$ from 1.33 to $0.53 \Omega$ and charge transfer resistances $\left(R_{\mathrm{CT}}\right)$ from 25.83 to $9.00 \Omega$. The decrease of $R_{\mathrm{S}}$ and $R_{\mathrm{CT}}$ is attributed to the existence of nitrogen in the PL-AC, resulting in a higher conductivity of electrode material and an enhancement of the charge transfer between electrode material and electrolyte. The large surface area of the PL- and CL-ACs was successfully achieved without detriment to the electrical conductivity. These results suggest that nitrogen-rich $\mathrm{PL}$ and $C L$ are potential precursors for the synthesis of nitrogen-doped AC in a one-step process, which can be used as an alternative electrode material for electrochemical capacitors and can potentially be applied for large-scale industrial production with low cost.

\section{Introduction}

Agriculture has long been the mainstay of the Thai economy, with abundant natural resources, and the majority of the Thai population engages in agricultural practices. The total area of Thailand is approximately $513000 \mathrm{~km}^{2}$ and $46 \%$ of this is used for agricultural purposes. After harvesting, there is a large amount of agricultural waste left. Thus, it is strongly desired to reduce the amount of agricultural waste and investigate the potential to maximize its uses. Agricultural waste can be used as feed for livestock, biomass energy, and raw material for activated carbon (AC) production. For use in AC, the raw materials are currently dominated by trunk, branch, shell, and seed of plants which are mainly composed of cellulose, lignin and

${ }^{a}$ College of Nanotechnology, King Mongkut's Institute of Technology Ladkrabang, Chalongkrung Road, Ladkrabang, Bangkok 10520, Thailand. E-mail: winadda.wo@ kmitl.ac.th

${ }^{b}$ Institute of Carbon Science and Technology, Shinshu University, 4-17-1 Wakasato, Nagano, 380-8553, Japan.E-mail: takeuchi@endomoribu.shinshu-u.ac.jp

'Interdisciplinary Graduate School of Science and Technology, Shinshu University, 417-1 Wakasato, Nagano, 380-8553, Japan hemicellulose. ${ }^{1-7}$ The obtained AC exhibits a high content of carbon with high surface area and high porosity. These ACs can be used in a wide application such as gas purification, metal extraction, water purification, sewage treatment, air filters and electrode in energy storage devices. Besides the above mentioned parts of the plants, there is a growing interest in the use of green leaves as precursors for the AC production. The green leaves contain nitrogen and a pigment called chlorophyll responsible for photosynthesis. The green leaves-derived AC is expected to contain nitrogen which is initially included in the raw materials, affording one-step preparation process of the nitrogen-doped AC. ${ }^{\mathbf{8 1 1}}$ The nitrogen-doped AC has the potential to broaden the appeal of $\mathrm{AC}$ in both existing and emerging applications, especially in the use as electrode materials of high-performance electrochemical capacitor (EC).

Recently, EC has been focused due to the increasing energy crisis arising from the limited fossil fuels. EC has the potential to bridge the gap between battery and conventional capacitor, as the great advantages including high power density, high rates of charge/discharge and long cycle life. ${ }^{12-16}$ To improve the performance of EC, most efforts have been focused on the improvement of electrode materials. AC is currently extensively 
examined and widely utilized as electrode materials with a focus on high specific surface area (SSA), hierarchical porous structure (combination of macro-, meso-, and micro-pores), and heteroatom doping (introduction of nitrogen $(\mathrm{N})$ and oxygen $(\mathrm{O})$ into the carbon framework). ${ }^{8,17-19}$ In addition, heteroatom doping can improve capacitance value due to charge transfer from faradic reaction as well as the increased accessibility of the electrolytes to electroactive species. ${ }^{\mathbf{8} 1 \mathbf{8 , 2 0 - 2 3}}$ The microstructure and the element composition of resulting AC largely depend on the raw materials. Thus, by selecting appropriate raw materials, the AC with a controlled microstructure and a desired amount of heteroatoms could be obtained.

In this study, a novel perspective of agricultural wastes for high-performance AC derived from green leaves, papaya and Coccinia grandis leaves (hereinafter referred to as PL and CL, respectively), is proposed. Papaya and Coccinia grandis are cultivated as an important food plant in many countries such as Mexico, India, Thailand, and Southeast Asia. The great popularity of papaya and Coccinia grandis may be attributed to several factors; it is easy to grow and has a high productivity. All parts of papaya tree and Coccinia grandis contain medicinal properties and are used by Thais as herbal medicine also. Synthesis of AC from PL and CL (hereinafter referred to as PL-AC and CL-AC, respectively) was demonstrated by carbonization at $400{ }^{\circ} \mathrm{C}$ followed with acid cleaning and $\mathrm{NaOH}$ activation. Surpassing the conventional bamboo-derived AC (hereinafter referred to as B$\mathrm{AC})$, the PL-and CL-ACs shows the hierarchical porous structure (combination of macro-, meso-, and micro-pores), high SSA, numerous heteroatom ( $\mathrm{O}$ and $\mathrm{N}$ ) groups, good electrical conductivity, and the wettability properties. To demonstrate their potential application as electrode material of the EC, the electrochemical properties of the PL- and CL-ACs were investigated. The specific capacitances of the PL- and CL-ACs were 98.47 and $89.91 \mathrm{~F} \mathrm{~g}^{-1}$, respectively, in aqueous electrolyte. The PL-AC shows the dramatic decrease in the series resistances $\left(R_{\mathrm{S}}\right)$ from 1.33 to $0.53 \Omega$ and the charge transfer resistances $\left(R_{\mathrm{CT}}\right)$ from 25.83 to $9.00 \Omega$. These results suggest that nitrogen-rich PL and CL are potential precursors for the synthesis of nitrogendoped AC in one-step process and can potentially be applied for large-scale industrial production with a low cost.

\section{Experimental}

\subsection{Preparation of activated carbon from papaya and Coccinia leaves}

The PL and CL were used as the precursors of the AC production. The fresh PL and CL were obtained from Thailand local farm. The typical AC synthesis process is shown in Fig. 1. Firstly, the fresh leaves were washed with deionized water and dried in an oven at $100{ }^{\circ} \mathrm{C}$ for $12 \mathrm{~h}$. The leaves were grinded into small pieces by a blender. The carbonization of the leaves was carried out at $400{ }^{\circ} \mathrm{C}$ for $2 \mathrm{~h}$ at a heating rate of $10^{\circ} \mathrm{C} \mathrm{min}{ }^{-1}$ under argon

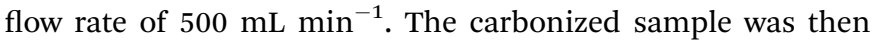
washed with $1 \mathrm{M}$ of sulfuric acid $\left(\mathrm{H}_{2} \mathrm{SO}_{4}\right)$ on a hotplate at $100{ }^{\circ} \mathrm{C}$ for $3 \mathrm{~h}$ to remove impurities, and was repeatedly washed with deionized water until neutral $\mathrm{pH}$. It was dried at $110{ }^{\circ} \mathrm{C}$ in ambient for $12 \mathrm{~h}$. The activation process was conducted by the heat-treatment of a solid mixture of carbonized sample and sodium hydroxide $(\mathrm{NaOH})$ (at the weight ratio of $1: 2.5$ ) in the tubular furnace at $720^{\circ} \mathrm{C}$ for $1 \mathrm{~h}$ under argon gas at a flow rate of $500 \mathrm{~mL} \mathrm{~min}^{-1}$. After that, the sample was washed with deionized water and dried under air ambient at $110{ }^{\circ} \mathrm{C}$ for overnight. The ACs thus derived from PL and CL are hereafter referred to as PL-AC and CL-AC, respectively. For comparison, the AC from the bamboo (hereafter referred to as $\mathrm{B}-\mathrm{AC}$ ) was prepared by the same processes.

\subsection{Material characterizations}

The morphologies of all the materials were characterized by scanning electron microscope (SEM, JEOL JSM-700F). The Raman spectra were observed by Raman spectroscope (Renishaw) using a laser wavelength of $532 \mathrm{~nm}$. The nitrogenadsorption isotherms were observed by the gas adsorption analyzer (Micromeritics ASAP 2020). The BET surface area and pore volume were determined from the adsorption isotherms. The calculation of pore volume was performed based on the method of density functional theory. ${ }^{24}$ The elemental and the chemical composition of the sample were estimated by X-ray photoelectron spectroscopy (XPS, PHI Quantera II).

\subsection{Characterization of electrochemical properties}

To characterize the electrochemical properties of the synthesized AC, the AC samples were used as the host material of the electrodes of coin type supercapacitor. To construct the electrode, each AC sample was mixed with carbon black and polytetrafluoroethylene (PTFE) in the weight ratio of $90: 5: 5$, respectively, in the mortar. Then, the mixture was pressed under applied pressure of $10 \mathrm{kN}$ for $1 \mathrm{~min}$ to form an electrode pellet with a diameter of $10 \mathrm{~mm}$, and subsequently dried at $100^{\circ} \mathrm{C}$ for $12 \mathrm{~h}$ in a vacuum oven. The total mass loading of the electrode material was approximately $20 \mathrm{mg}$. Two types of electrolytes were used to characterize the electrochemical properties; $1 \mathrm{M} \mathrm{Na}_{2} \mathrm{SO}_{4}$ as an aqueous electrolyte and $1 \mathrm{M}$ tetraethylammonium tetrafluoroborate $\left(\mathrm{Et}_{4} \mathrm{NBF}_{4}\right)$ dissolved in propylene carbonate as an organic electrolyte. In the case of the aqueous electrolyte, the electrochemical performance was characterized using a conventional Teflon electrochemistry cell with a three-electrode cell, consisting of two identical electrode pellets on $\mathrm{Ni}$ foams with a relatively same weight separated by the a $30 \mu \mathrm{m}$-thick separator serving as working and counter electrodes, and a $\mathrm{Ag}$ wire as the reference electrode. In the case of the organic electrolyte, the electrochemical performance was determined using a conventional two-electrode coin cell, consisting of two identical electrode pellets with a relatively same weight separated by the a $30 \mu \mathrm{m}$-thick separator. All electrochemical tests were carried out by an electrochemical workstation (Bio-Logic Science Instruments VSP Potentiostat). The electrochemical properties were characterized by cyclic voltammetry (CV), galvanostatic charge-discharge (CD), and electrochemical impedance spectroscopy (EIS, with an amplitude of $5 \mathrm{mV}$ in the frequency range of $1 \mathrm{MHz}$ to $10 \mathrm{mHz}$ ). The specific capacitances of the supercapacitor $\left(C_{\mathrm{S}}\right)$ were evaluated from $\mathrm{CD}$ curves using the following equations: 


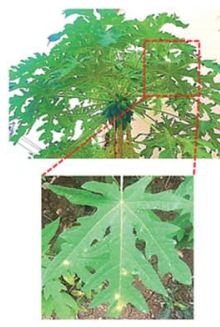

Papaya

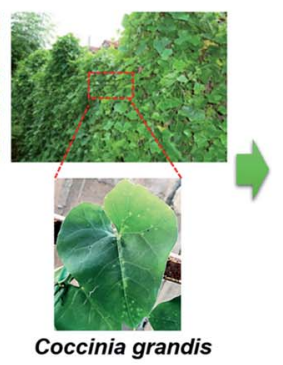

Coccinia grandis

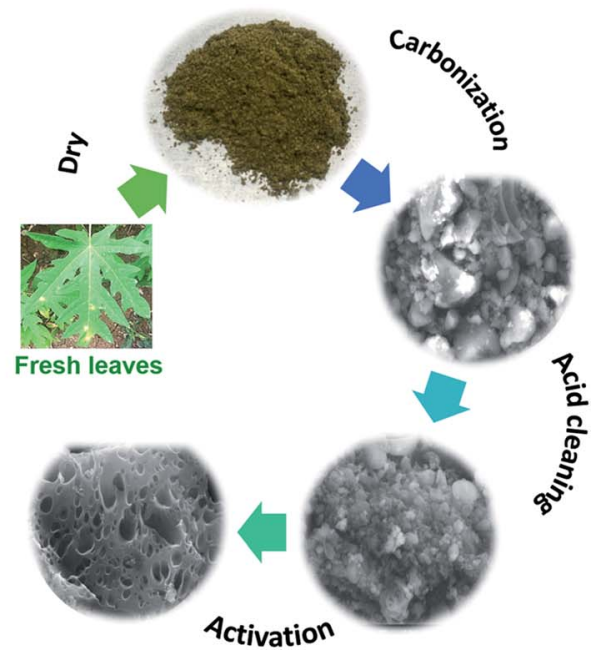

Fig. 1 Schematic illustration of activated carbon (AC) synthesis.

$$
C_{\mathrm{S}}=\frac{I \times \Delta t}{\Delta V \times m}
$$

where $C_{\mathrm{S}}, I, \Delta t, \Delta \mathrm{V}$ and $m$ are the specific capacitance $\left(\mathrm{F} \mathrm{g}^{-1}\right)$, discharge current (A), discharge time (s), voltage change after a full charge or discharge (V) and the total loading mass of two electrode pellets $(\mathrm{g})$, respectively.

\section{Results and discussion}

\subsection{Morphology and structure characterization}

Fig. 2 shows SEM images at low and high magnifications of the PL-AC (Fig. 2a and d), CL-AC (Fig. 2b and e), and B-AC (Fig. 2c and f), respectively. The morphologies of three types of AC were totally different. The PL-AC shows a sponge-like structure, with lots of pores, while the CL-AC contains many small wrinkled sheets fused each other. The B-AC shows the coagulation of fibrous and clustered particles. Fig. 3a shows the nitrogen adsorption and desorption isotherms at $77 \mathrm{~K}$ of each sample. The adsorption of nitrogen was remarkably increased in the relatively low pressure region and then gradually saturated. In addison, hysteresis was not obviously observed. The PL-AC shows the highest saturated adsorption amount. Thus, the AC samples can be classified as the type I isotherms (definition of IUPAC $^{25}$ ), implying the existence of the microporous solids. Fig. 3b shows the pore size distribution of the AC samples obtained by the DFT method. It can be seen that all the samples have pore sizes mainly in the range of $0.5-3.7 \mathrm{~nm}$, in which the
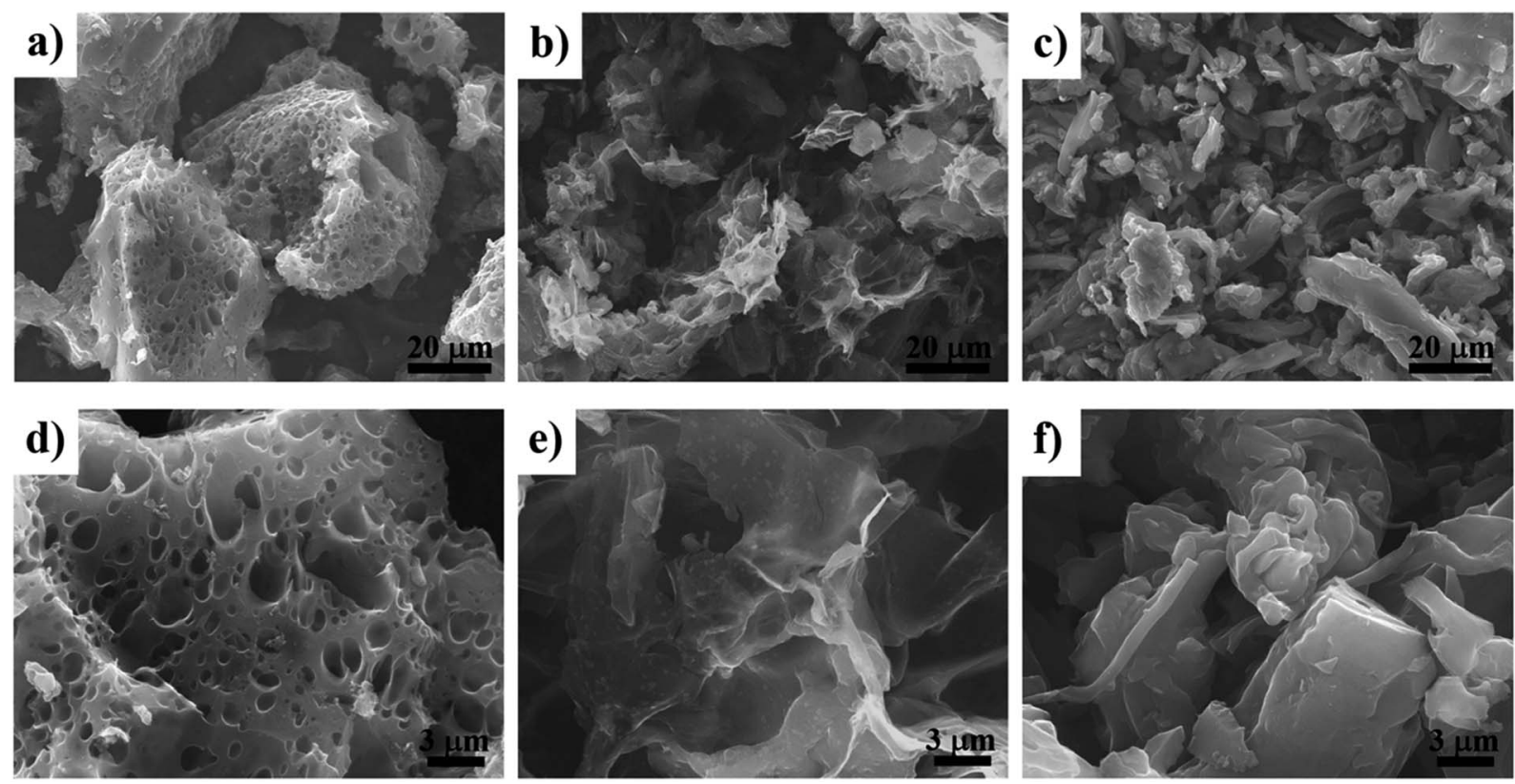

Fig. 2 SEM images at low and high magnifications: (a) and (d), PL-AC; (b) and (e) CL-AC; (c) and (f), B-AC. 

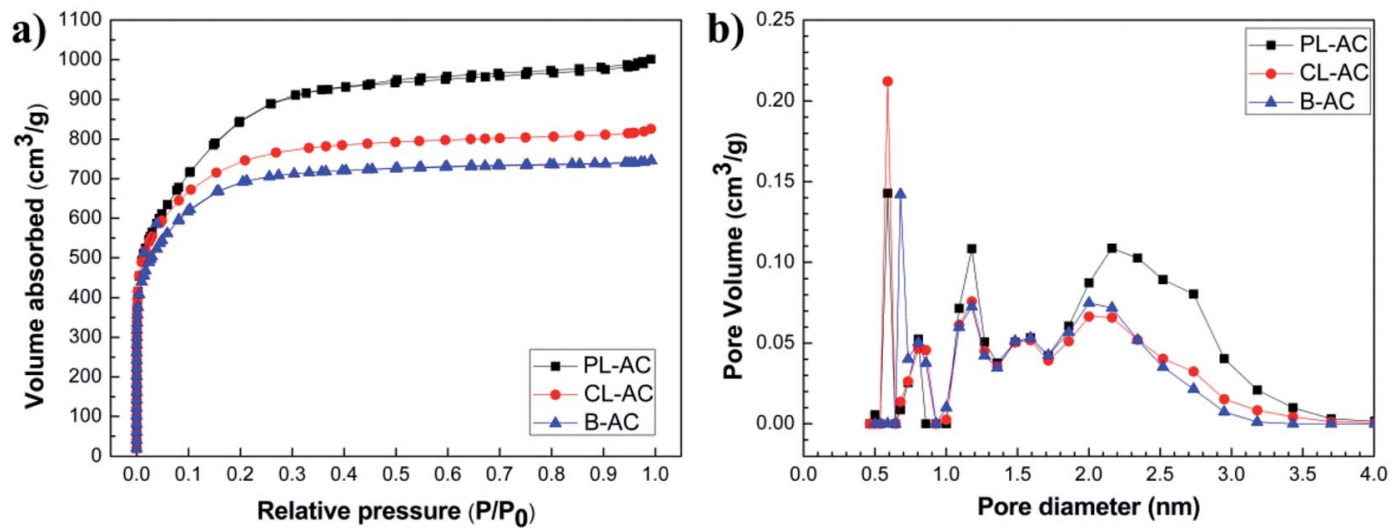

Fig. 3 (a) Nitrogen adsorption and desorption isotherms at $77 \mathrm{~K}$, and (b) pore size distribution of PL-, CL- and B-ACs.

Table 1 Surface properties of ACs estimated from $\mathrm{N}_{2}$ adsorption/desorption isotherms

\begin{tabular}{|c|c|c|c|c|c|c|c|}
\hline Sample & $S_{\mathrm{BET}}^{a}\left(\mathrm{~m}^{2} \mathrm{~g}^{-1}\right)$ & $V_{\text {total }}^{b}\left(\mathrm{~cm}^{3} \mathrm{~g}^{-1}\right)$ & $V_{\text {micro }}{ }^{c}\left(\mathrm{~cm}^{3} \mathrm{~g}^{-1}\right)$ & $V_{\text {meso }}{ }^{d}\left(\mathrm{~cm}^{3} \mathrm{~g}^{-1}\right)$ & $V_{\text {macro }}^{e}\left(\mathrm{~cm}^{3} \mathrm{~g}^{-1}\right)$ & $V_{\text {micro }} / V_{\text {total }}(\%)$ & $V_{\text {meso }} / V_{\text {total }}(\%)$ \\
\hline CL-AC & 2576 & 1.054 & 0.824 & 0.221 & 0.008 & 78.18 & 20.96 \\
\hline
\end{tabular}

${ }^{a}$ BET specific surface area. ${ }^{b}$ DFT total pore volume. ${ }^{c}$ DFT micro-pore volume. ${ }^{d}$ DFT meso-pore volume. ${ }^{e}$ DFT macro-pore volume.

PL- and CL-ACs possess the highest pore volume at the diameter of $0.6 \mathrm{~nm}$, in comparison with that at slightly larger diameter of $0.7 \mathrm{~nm}$ for B-AC. The specific surface area $\left(S_{\mathrm{BET}}\right)$ and pore volume of all the samples calculated from nitrogen adsorption/ desorption isotherms were summarized in Table 1. The PL-AC has the highest $S_{\mathrm{BET}}\left(2664 \mathrm{~m}^{2} \mathrm{~g}^{-1}\right)$ and $V_{\text {total }}\left(1.27 \mathrm{~cm}^{3} \mathrm{~g}^{-1}\right)$, whereas the B-AC has the lowest $S_{\mathrm{BET}}\left(2370 \mathrm{~m}^{2} \mathrm{~g}^{-1}\right)$ and $V_{\text {total }}$ $\left(0.97 \mathrm{~cm}^{3} \mathrm{~g}^{-1}\right)$. The $S_{\mathrm{BET}}$ and $V_{\text {total }}$ of the CL-AC are $2576 \mathrm{~m}^{2} \mathrm{~g}^{-1}$

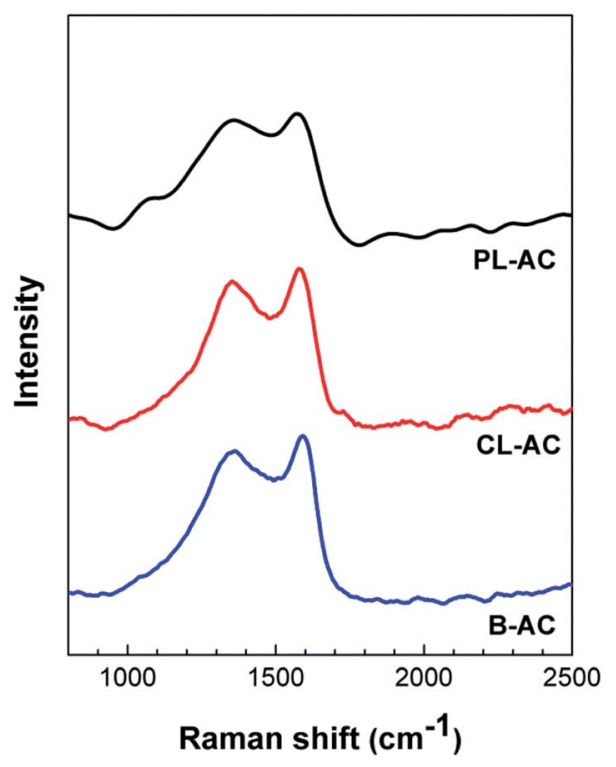

Fig. 4 Raman spectra of PL-, CL- and B-ACs. and $1.05 \mathrm{~cm}^{3} \mathrm{~g}^{-1}$, respectively. The PL-AC is characterized by the relatively high $V_{\text {micro }}\left(0.798 \mathrm{~cm}^{3} \mathrm{~g}^{-1}\right)$ and the highest $V_{\text {meso }}$ $\left(0.466 \mathrm{~cm}^{3} \mathrm{~g}^{-1}\right.$ with pore sizes ranging from 2.0 to $\left.3.7 \mathrm{~nm}\right)$. The ratios of the $V_{\text {micro }}$ to $V_{\text {total }}$ and the $V_{\text {meso }}$ to $V_{\text {total }}$ of the PL-AC are 62.83 and $36.69 \%$, respectively. The existence of mesopores in the AC provides a favorable path for transportation and penetration of electrolyte ion, which is important for fast ion transfer. ${ }^{19}$

Fig. 4 shows Raman spectra of the AC samples. All the AC samples exhibit two broad overlapping bands, which are the socalled D-band originated from the defects and disordered carbon structure at a Raman shift of around $1360 \mathrm{~cm}^{-1}$, and the G-band originated from the graphitic structure at a Raman shift of around $1600 \mathrm{~cm}^{-1} \cdot{ }^{26,27}$ The ratio of the intensities of the D peak to the $G$ peak ( $D / G$ ratio) is generally used as the measure of the degree of graphitization of activated carbon, and the higher ratio means the lower degree of graphitization. ${ }^{27}$ The corresponding values of the $\mathrm{D} / \mathrm{G}$ ratio were $1.00,0.988$, and 0.941 for the PL-, CL- and B-AC, respectively. The high D/G ratio indicates the less graphitized carbons. ${ }^{28,29}$ It is known that the introduction of nanoscale pores on carbon framework usually leads to an increase of defects and disorders. ${ }^{30}$ Thus, the relatively high D/G ratio of the PL- and CL-ACs implies the structural defects are concerned with the large pore volume in the PL- and CL-ACs. The above results confirms that the PL and CL can be used as the promising precursors to obtain the AC containing nanoporous carbon with relatively large specific surface area and micro-pore volume surpassing the B-AC and the commercial AC (Kansai Coke and Chemicals Company). ${ }^{7}$ 


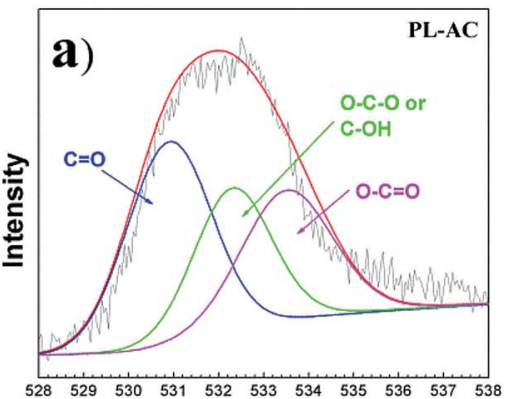

Binding energy (eV)

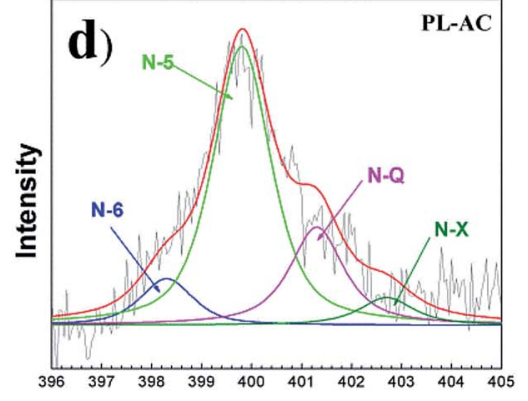

Binding energy (eV)

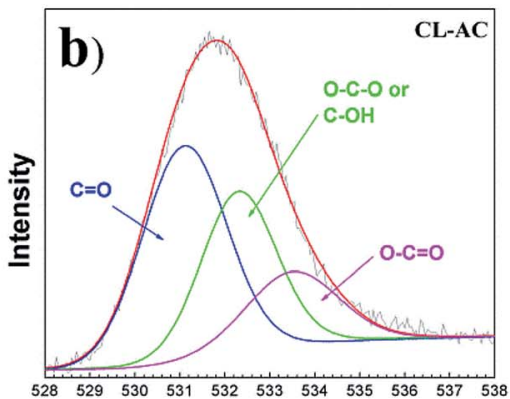

Binding energy (eV)

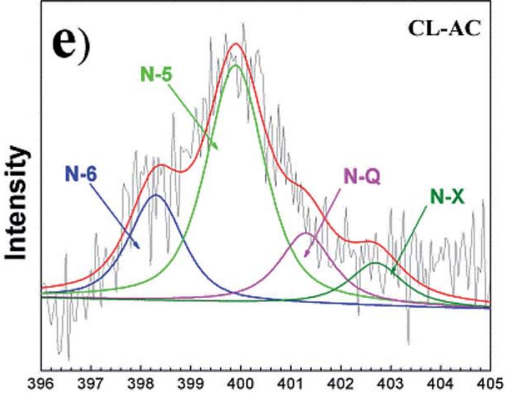

Binding energy $(\mathrm{eV})$

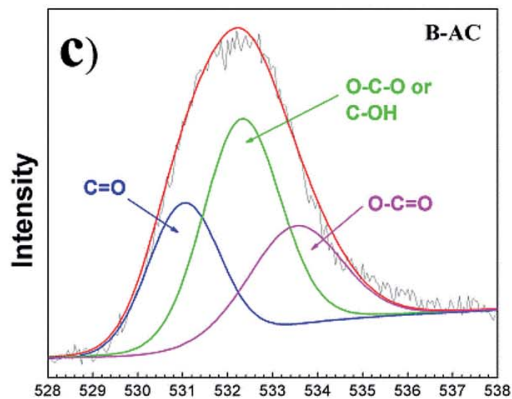

Binding energy (eV)

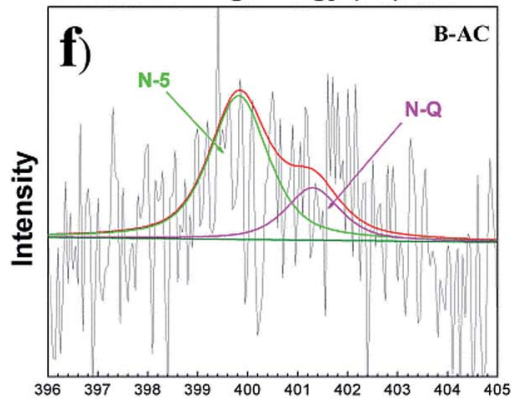

Binding energy (eV)

Fig. $5 \mathrm{O}$ 1s and N 1s XPS spectra of PL-, CL- and B-ACs: (a) and (d), PL-AC; (b) and (e), CL-AC; (c) and (f), B-AC.

\subsection{Elemental and chemical composition}

The chemical composition of the AC samples was studied by XPS. Fig. 5 shows XPS spectra of $\mathrm{O} 1 \mathrm{~s}$ and $\mathrm{N}$ 1s observed for the AC samples. The chemical composition, N/C ratio and the relative $\mathrm{N}$ 1s peak area are summarized in Table 2. The PL-AC shows the highest nitrogen content (2.3 at\%) and the N/C ratio (0.025), while the CL-AC shows the highest oxygen content of $9.5 \mathrm{at} \%$. The PLand CL-ACs show relatively high nitrogen content compared to that of the B-AC, implying that nitrogen-rich leaves can be used as precursors of the nitrogen-doped AC. The high-resolution $\mathrm{O} 1 \mathrm{~s}$ spectra, shown in Fig. $5 \mathrm{a}-\mathrm{c}$, can be divided into three different binding configurations, including $\mathrm{O}-\mathrm{C}=\mathrm{O}$ in carboxylic (533.5 $\mathrm{eV}), \mathrm{O}-\mathrm{C}-\mathrm{O} / \mathrm{C}-\mathrm{OH}$ in ester or phenol $(532.2 \mathrm{eV})$, and $\mathrm{C}=\mathrm{O}$ in carbonyl/quinone $(531.2 \mathrm{eV}) .^{18,31}$ The content of oxygen functional groups included in each AC is different. The dominant oxygen functional group in the PL- and CL-ACs is $\mathrm{C}=\mathrm{O}$, while that of the $\mathrm{B}-\mathrm{AC}$ is $\mathrm{O}-\mathrm{C}-\mathrm{O} / \mathrm{C}-\mathrm{OH}$. On the other hand, the high-resolution $\mathrm{N} 1 \mathrm{~s}$ spectra, shown in Fig. 5d-f, can be deconvoluted by incorporation

Table 2 Chemical composition of PL-, CL- and B-ACs evaluated from elemental analysis

\begin{tabular}{|c|c|c|c|c|c|c|c|c|}
\hline \multirow[b]{2}{*}{ Sample } & \multicolumn{3}{|c|}{ Content (at\%) } & \multirow[b]{2}{*}{$\mathrm{N} / \mathrm{C}$ ratio } & \multicolumn{4}{|c|}{ Relative N 1s peak area $^{a}(\%)$} \\
\hline & $\mathrm{C}$ & $\mathrm{O}$ & $\mathrm{N}$ & & $\mathrm{N}-6$ & N-5 & $\mathrm{N}-Q$ & $\mathrm{~N}-X$ \\
\hline PL-AC & 91.0 & 6.6 & 2.3 & 0.025 & 0.21 & 1.44 & 0.50 & 0.14 \\
\hline CL-AC & 87.3 & 9.5 & 1.8 & 0.021 & 0.39 & 0.95 & 0.28 & 0.17 \\
\hline B-AC & 89.3 & 9.1 & 0.1 & 0.001 & - & 0.07 & 0.03 & - \\
\hline
\end{tabular}

${ }^{a}$ Pyridine $\mathrm{N}(\mathrm{N}-6)$, pyrrolic $\mathrm{N}(\mathrm{N}-5)$, quaternary $\mathrm{N}(\mathrm{N}-\mathrm{Q})$ and oxidized pyridine $\mathrm{N}(\mathrm{N}-\mathrm{X})$. of four contributions including pyridine $\mathrm{N}(398.3 \mathrm{eV}, \mathrm{N}-6)$, pyrrolic $\mathrm{N}(399.89 \mathrm{eV}, \mathrm{N}-5)$, quaternary $\mathrm{N}(401.3 \mathrm{eV}, \mathrm{N}-Q)$ and oxidized pyridine $\mathrm{N}(402.70 \mathrm{eV}, \mathrm{N}-X) . .^{18,31,32}$ The content of nitrogen functional groups included in the green leaves-derived ACs and the B-AC are completely different. The PL- and CL-ACs show the compositions of $\mathrm{N}-6, \mathrm{~N}-5, \mathrm{~N}-Q$ and $\mathrm{N}-X$, with the highest content of $\mathrm{N}-5$, while the B-AC shows only the compositions of $\mathrm{N}-5$ and $\mathrm{N}-\mathrm{Q}$. Normally, N-5 and N-6 are the defects in the graphene layer and could induce pseudocapacitance property. In addition, high content $\mathrm{N}-Q$ group in $\mathrm{AC}$ obviously improve the electronic conductivity and the high content of $\mathrm{N}-X$ implies the wettability properties. These results show that the raw materials of AC directly affect the microstructure and heteroatom doping properties. Owing to the hierarchical porous structure (combination of macro-, meso-, and micro-pores), high SSA, numerous heteroatom $(\mathrm{O}$ and $\mathrm{N})$ groups, good electrical conductivity, and the wettability properties of the PL-and CL-ACs which are important requirements of an ideal electrode material of EC, it is a challenge to investigate the potential of the PL-and CL-ACs as the electrode material of EC.

\subsection{Electrochemical properties in aqueous electrolyte}

The electrochemical properties of the AC samples were investigated in a three-electrode system using $1 \mathrm{M} \mathrm{Na}_{2} \mathrm{SO}_{4}$ aqueous electrolyte. The cyclic voltammetry (CV) curves collected at a scan rate $1 \mathrm{mV} \mathrm{s}^{-1}$, and galvanostatic charge-discharge (CD) at an applied constant current of $5 \mathrm{~mA}$ are presented in Fig. $6 \mathrm{a}$ and b, respectively. The $\mathrm{CV}$ curves in a potential window from -1.0 to $0 \mathrm{~V}$ present quasi-rectangular shapes with obvious reversible hump, suggesting the capacitive response combination between the electrical double-layer capacitor (EDLC) and pseudo- 


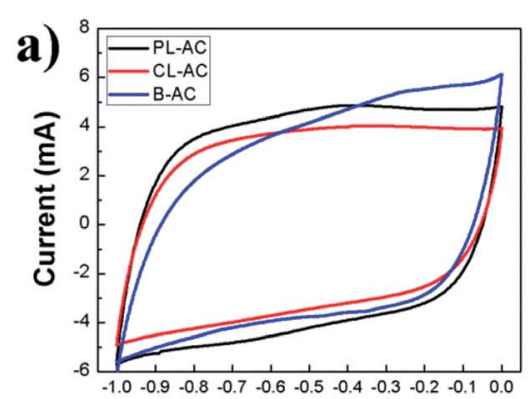

Potential (V)
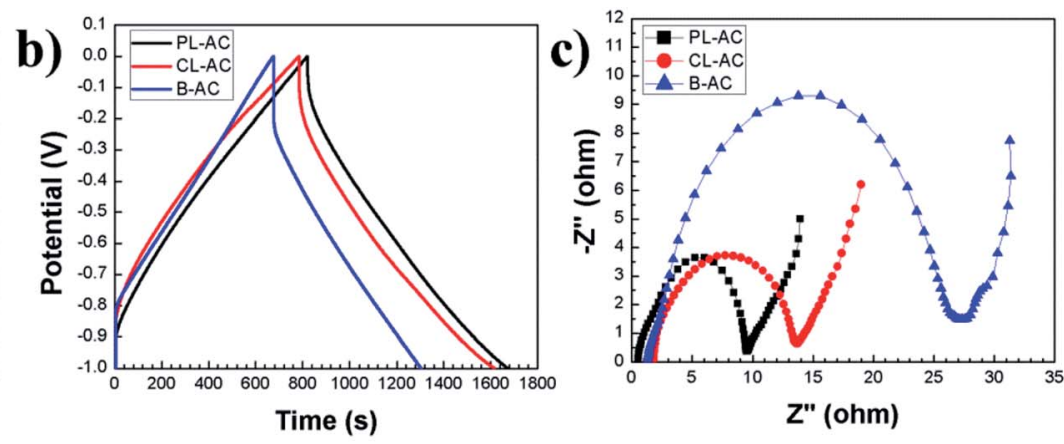

Fig. 6 Electrochemical properties of the PL-, CL- and B-ACs in $1 \mathrm{M} \mathrm{Na}_{2} \mathrm{SO}_{4}$ electrolyte from -1 to $0 \mathrm{~V}$ : (a), cyclic voltammetry curve at a scan rate $1 \mathrm{mV} \mathrm{s}^{-1}$; (b), galvanostatic charge-discharge curve of papaya electrode material at an applied constant current of $5 \mathrm{~mA}$; (c), Nyquist plot in the frequency range of $1 \mathrm{MHz}$ to $10 \mathrm{mHz}$ at an amplitude of $5 \mathrm{mV}$.

capacitor. The PL-AC exhibits the largest area of CV curve, indicating the highest capacitance due to the high specific surface area and the high nitrogen content. The steep slopes of the current change at the switching potential at a potential of $-0.7 \mathrm{~V}$ indicate a small mass-transfer resistance of all electrodes. Obviously, the hierarchical pore structure of all materials provides a short ion pathway and electrolyte reservoir for rapid ionic transportation. The CD curves (Fig. 6b) show a shape of an imperfectly symmetrical triangle, indicating that all AC samples have good electrochemical behaviors accompanied by the pseudocapacitive contribution of the functional group. From CD curves, the specified capacitances of the PL-, CL- and B-ACs were approximately $98.47,89.91$, and $83.68 \mathrm{~F} \mathrm{~g}^{-1}$, respectively. The excellent capacitive behavior of the PL-AC-based EC was also reflected in their long charge-discharge time. The best electrochemical performance of the PL-AC is attributed to the cocontribution of the high electronic double-layer capacitance due to its high surface area and the additional pseudocapacitance mainly derived from the doped nitrogen. To further understand the origin of the improved electrochemical performance of the PL-AC, the EIS characteristics were investigated.

Fig. 6c shows the Nyquist plots of all AC samples in the frequency range of $1 \mathrm{MHz}$ to $10 \mathrm{mHz}$ at an amplitude of $5 \mathrm{mV}$. The EIS spectra consist of a semicircle in the high-frequency and a linear line in the low-frequency regions. All AC samples show resembles semicircle curve, but different offsets on the $x$-axis and different diameters, revealing their different characteristics. The offset on the $x$-axis $\left(Z^{\prime}\right)(\Omega)$ of the high-frequency semicircle is a series resistance $\left(R_{\mathrm{S}}\right)$ of the electrode consisting of the electrode material resistance, the electrolyte resistance and the contact resistance between the electrode material and the current collector. The values of $R_{\mathrm{S}}$ of the PL-, CL- and B-ACs are estimated to be $0.53,1.82$, and $1.33 \Omega$, respectively. The diameter of the semicircle represents the kinetic resistance to the charge transfer, called the charge transfer resistance $\left(R_{\mathrm{CT}}\right)$. The values of $R_{\mathrm{CT}}$ of the PL-, CL- and B-ACs are estimated to be $9.00,11.86$, and $25.83 \Omega$, respectively. In the case of $\mathrm{AC}$, its properties in terms of surface area, pore structure and conductivity need to be monitored as these properties can be mutually exclusive, that is, one can be improved only to the detriment of another. In this experiment, the significant decrease in $R_{\mathrm{S}}$ and $R_{\mathrm{CT}}$ of the PL-AC although its relatively large surface area compared to that of the B-AC can be ascribed to the combination properties of high surface area, hierarchical porous structure, and nitrogen doping. In respect of the low value of $R_{\mathrm{S}}$, it may be attributed to the $\mathrm{N}-\mathrm{Q}$ existence. The quaternary $\mathrm{N}$ atoms take the position inside an aromatic ring with $\mathrm{sp}^{2}$ hybridization and fully saturated bonding, and further enhance the electrical conductivity of the carbon material. ${ }^{33}$ This is evidenced by the high content of $\mathrm{N}-Q$ in the PL-AC compared to the B-AC as shown in Table 2, resulting in the low $R_{\mathrm{S}}$ of the PL-AC although its high surface area. In respect of the value of $R_{\mathrm{CT}}$, it can be attributed to the following reasons: (i) nanoporous carbon of electrode materials. The large volume of the mesopore of the PL-AC compared to that of the CL- and BACs leads provide the accessible route to electrolyte to enter into electrode more easily. ${ }^{34}$ (ii) the existence of negatively charged nitrogen-containing groups of N-5 and N-6. Generally, $\mathrm{N}-5$ and N-6 species lie at the edges of graphitic layer, where nitrogen atom is bonded to two carbon atoms, creating cavities and defects. The nitrogen atom of N-6 contributes one $\pi$-electron to the aromatic ring and has a lone electron pair in the plane of ring, whereas the nitrogen atom of $\mathrm{N}-5$ contributes two $\pi$-electron and a hydrogen atom is bound in the plane of the ring: ${ }^{35,36}$ Due to the high electron density, $\mathrm{N}-5$ and N-6 act as the acceptor and can accept the donors, thereby causing the faradic reaction for contributing to pseudocapacitance (as expressed by $\mathrm{e}^{-}$with the curved blue arrows in Fig. 8). This is evidenced by the high content of N-5 and N-6 in the PL- and CL-ACs compared to the B-AC as shown in Table 2. (iii) The existence of the positively charged nitrogen-containing groups of $\mathrm{N}-\mathrm{Q}$ and $\mathrm{N}-X$. The $\mathrm{N}-Q$ and $\mathrm{N}-X$ are the nitrogen species that the nitrogen atom substitutes carbon atom in aromatic ring, and the oxidized pyridine nitrogen atom, respectively, providing the positively charge. Since fast electron transfer is a crucial requirement to keep the constant energy at any operation load. Most of electrochemical capacitors based on porous carbon often suffer from a capacitance drop caused by an increase in the ohmic resistance of porous carbon. Thus, the enrichment of carbons with positively charged $\mathrm{N}-Q$ and $\mathrm{N}-X$ may help in 
electron transfer through the porous carbon, resulting in enhancing the capacitance (as expressed by $\mathrm{e}^{-}$with the straight yellow arrows in Fig. 8). ${ }^{18}$ This is evidenced by the high content of $\mathrm{N}-Q$ and $\mathrm{N}-X$ in the PL- and CL-ACs compared to the B-AC as shown in Table 2. (iv) The existence of oxygen-containing functional groups. Generally, the micropores of carbon cannot be fully wetted in aqueous electrolyte, and thus are not fully accessible to electrolyte. ${ }^{37}$ Oxygen-containing functional groups such as $\mathrm{C}=\mathrm{O}$ and/or $\mathrm{C}-\mathrm{OH}$, and the oxidized pyridine $(\mathrm{N}-\mathrm{X})$ may improve carbon wettability of the internal structure of micropores, thus resulting in a corresponding decrease in $R_{\mathrm{CT}}$ and increase in specific capacitance (as expressed by ions of aqueous electrolyte in Fig. 8). The B-AC could also deliver the relatively good capacitance owing to the existence of the oxygencontaining functional groups and thus compensate the low concentration of nitrogen-containing groups and its relatively low surface area.

\subsection{Electrochemical properties in organic electrolyte}

To investigate the versatile use of the green leaves-derived AC in various types of electrolyte, the electrochemical performance of the AC samples were also characterized in the organic electrolyte in order to achieve higher operational voltages and therefore greater specific energy. Similar to the case of aqueous electrolyte, the rectangular $\mathrm{CV}$ curves at a scan rate $1 \mathrm{mV} \mathrm{s}^{-1}$ in a potential window from 0 to $2.5 \mathrm{~V}$ were obtained confirming the electrochemical properties (Fig. 7a). The imperfectly symmetrical triangle of the CD curves in an applied constant current of $5 \mathrm{~mA}$ shown in Fig. 7b also indicates their electrochemical properties. From the Nyquist plot shown in Fig. 7c, the PL-, CL- and B-ACs show the $R_{\mathrm{S}}$ of $1.05,1.27$ and $2.40 \Omega$, respectively, with the $R_{\mathrm{CT}}$ of $13.60,23.06$ and $44.02 \Omega$, respectively. The increase in the $R_{\mathrm{S}}$ and $R_{\mathrm{CT}}$ in the organic electrolyte compared to that of aqueous electrolyte is due to the lower ionic conductivity of the organic electrolyte. Nevertheless, the PL-AC shows the smallest $R_{\mathrm{S}}$ and $R_{\mathrm{CT}}$, especially with the 3.4-fold lowering in $R_{\mathrm{CT}}$ compared to that of the B-AC. The specific capacitances calculated from the charge-discharge measurement of all AC samples at different current densities ranging from 0.02 to $0.5 \mathrm{Ag}^{-1}$ are shown in Fig. 7d. The PL-AC shows the highest specified capacitances of $47 \mathrm{~F} \mathrm{~g}^{-1}$ at the current density of $0.02 \mathrm{~A} \mathrm{~g}^{-1}$ with the relatively stable specific capacitance at the high current density up to $0.5 \mathrm{~A} \mathrm{~g}^{-1}$, implying the relatively high capacitance retention of $95.65 \%$. The specific capacitances of the CL- and B-ACs are 45 and $42 \mathrm{~F} \mathrm{~g}^{-1}$, respectively. Moreover, the energy density and the power density were calculated at the current density of $0.5 \mathrm{~A} \mathrm{~g}^{-1}$. The energy density of the PL-, CL- and B-ACs was approximately 39, 33 and $30 \mathrm{~W} \mathrm{~h} \mathrm{~kg}{ }^{-1}$, respectively, while the power density of the PL-, CL- and B-ACs was approximately $1.7,3.3$ and $4.4 \mathrm{~kW} \mathrm{~kg}^{-1}$, respectively.

Fig. 7e shows the specific capacitance $v s$. the cycle numbers of CD cycles at the constant current of $10 \mathrm{~mA}$ of all AC samples. The specific capacitance of the PL-, CL- and B-ACs remains 98.70, 95.02 and 91.26\% after 3500 CD cycles. All AC samples
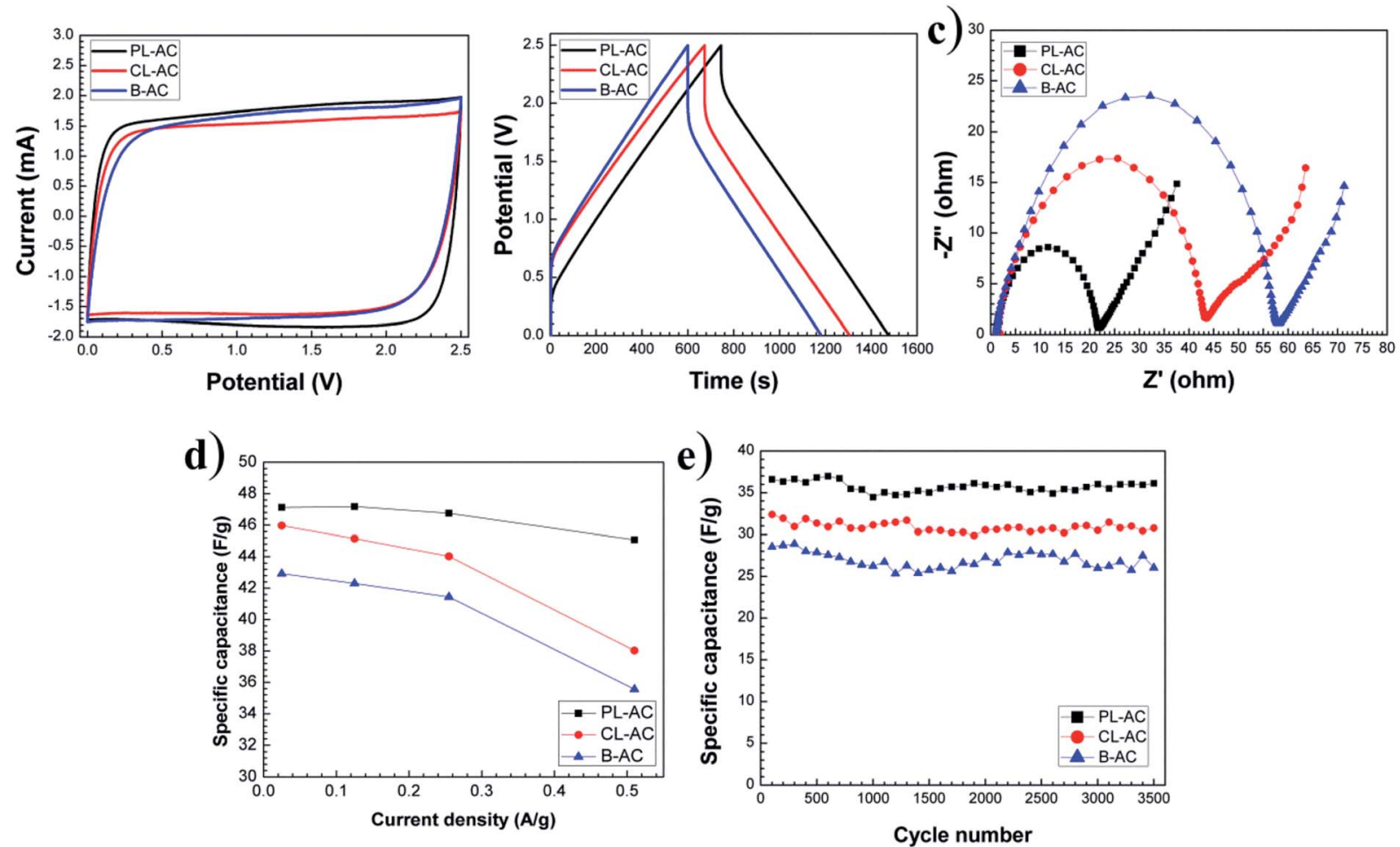

Fig. 7 Electrochemical properties of the PL-, CL- and B-ACs in $1 \mathrm{M} \mathrm{Et}_{4} \mathrm{NBF}_{4}$ electrolyte from 0 to $2.5 \mathrm{~V}$ : (a), cyclic voltammetry curve at a scan rate $1 \mathrm{mV} \mathrm{s}^{-1}$; (b), galvanostatic charge-discharge curve at an applied constant current of $5 \mathrm{~mA}$; (c), Nyquist plot in the frequency range of $1 \mathrm{MHz}$ to $10 \mathrm{mHz}$ at an amplitude of $5 \mathrm{mV}$; (d), dependence of specific capacitance on current density from 1.27 to $25.46 \mathrm{~mA} \mathrm{~cm}^{-2}$; (e), cycling stability at an applied constant current of $10 \mathrm{~mA}$. 


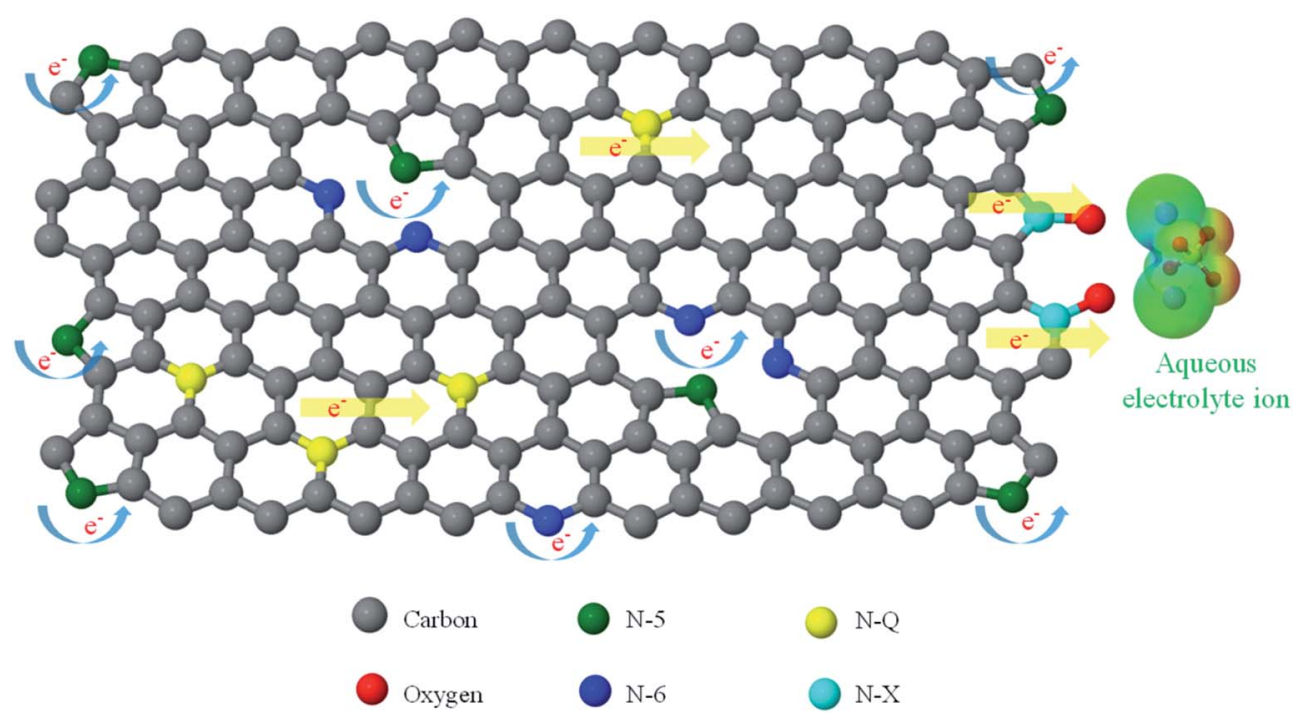

Fig. 8 Schematic view of the effect of the properties of the $N-5, N-6, N-Q$ and $N-X$ on the charge transfer resistance.

show good stability with good retention of capacitance. The specific capacitance, energy density and power density of the PL-AC is relatively comparable to AC derived from other parts of the tree. ${ }^{8,9,11,38,39}$ In our study, not only the specific surface area of the activated carbon, the effect of the role of heteroatom doping (nitrogen and oxygen) on electrochemical properties also discussed. The morphology and structure of the PL-AC can be further maximized by using the optimized activation agent such as the mixing of $\mathrm{K}_{2} \mathrm{CO}_{3}$ and $\mathrm{KOH}$, which is importance in enlargement of pore channel, providing the relatively high specific surface area. ${ }^{17}$

\section{Conclusion}

In summary, the nitrogen-containing ACs derived from the green leaves of PL and CL were successfully synthesized by carbonization, acid cleaning and chemical activation processes. The PL- and CL-ACs show the relatively high specific surface area of 2664 and $2576 \mathrm{~m}^{2} \mathrm{~g}^{-1}$, respectively, compared to that of the B-AC $\left(2370 \mathrm{~m}^{2} \mathrm{~g}^{-1}\right)$. The nitrogen contents composing of N$6, \mathrm{~N}-5, \mathrm{~N}-Q$ and $\mathrm{N}-X$ including in the PL- and CL-ACs was approximately 2.3 and 1.8 at\%, respectively. Thus, the PL- and CL-ACs offer the relatively high specific surface area, hierarchical porous structure (combination of macro-, meso-, and micro-pores), good conductivity and heteroatom doping, fulfilling the requirement of the use as the electrode material of AC. The PL- and CL-ACs shows excellent electrochemical properties in both aqueous and organic electrolytes. The specific capacitances of the PL- and CL-ACs were 98.47 and $89.91 \mathrm{~F} \mathrm{~g}^{-1}$, respectively, comparable to $83.68 \mathrm{~F} \mathrm{~g}^{-1}$ of the B-AC in $\mathrm{Na}_{2} \mathrm{SO}_{4}$ electrolyte. Especially, compared to the B-AC, the PL-AC shows the dramatic decrease in the series resistances $\left(R_{\mathrm{S}}\right)$ from 1.33 to $0.53 \Omega$ and the charge transfer resistances $\left(R_{\mathrm{CT}}\right)$ from $25.83 \Omega$ to $9.00 \Omega$. The decrease of $R_{\mathrm{S}}$ and $R_{\mathrm{CT}}$ is attributed to the existence of the nitrogen in the PL-AC, resulting in a higher conductivity of electrode material and an enhancement of the charge transfer between electrode material and electrolyte. These results suggest that nitrogen-rich PL and CL are potential precursors for the synthesis of nitrogen-doped activated carbon by one-step process, and can potentially be applied for largescale industrial production with a low cost.

\section{Contributions}

Synthesis and characterizations were done by P. D., M. F., I. Y., Y. T., Y. B, Y. T., T. A. and M. P. Manuscript was written by P. D., M. F., W. W., K. T. and M. E. with discussions.

\section{Conflicts of interest}

There are no conflicts to declare.

\section{Acknowledgements}

This research was partially supported by grants from the Project of the NARO Bio-oriented Technology Research Advancement Institution (Integration research for agriculture and interdisciplinary fields). We also thank Dr Noboru Akuzawa of Shinshu University for his kind suggestions. We also acknowledge the support from the Strategic Scholarships Fellowships Frontier Research Networks (Specific for Southern region) from the Office of the Higher Education Commission (OHEC).

\section{References}

1 J. Deng, T. Xiong, H. Wang, A. Zheng and Y. Wang, ACS Sustainable Chem. Eng., 2016, 4, 3750-3756.

2 M. Z. Hussein, M. B. Abdul Rahman, A. H. Yahaya, Y. H. Taufiq-Yap and N. Ahmad, J. Porous Mater., 2001, 8, 327-334. 
3 A. Jain, V. Aravindan, S. Jayaraman, P. S. Kumar, R. Balasubramanian, S. Ramakrishna, S. Madhavi and M. P. Srinivasan, Sci. Rep., 2013, 3, 1-6.

4 M. Dhelipan, A. Arunchander, A. K. Sahu and D. Kalpana, J. Saudi Chem. Soc., 2016, 21, 487-494.

5 R. K. Gupta, M. Dubey, P. Kharel, Z. Gu and Q. H. Fan, J. Power Sources, 2015, 274, 1300-1305.

6 S. Uçar, M. Erdem, T. Tay and S. Karagöz, Appl. Surf. Sci., 2009, 255, 8890-8896.

7 M. Fujishige, I. Yoshida, Y. Toya, Y. Banba and K. Oshida, Biochem. Pharmacol., 2017, 5, 1801-1808.

8 G. Ma, J. Li, K. Sun, H. Peng, E. Feng and Z. Lei, J. Solid State Electrochem., 2017, 21, 525-535.

9 B. Liu, Y. Liu, H. Chen, M. Yang and H. Li, J. Power Sources, 2017, 341, 309-317.

10 X. Xia, H. Liu, L. Shi and Y. He, J. Mater. Eng. Perform., 2012, 21, 1956-1961.

11 H. Peng, G. Ma, K. Sun, Z. Zhang, Q. Yang and Z. Lei, Electrochim. Acta, 2016, 190, 862-871.

12 P. Simon and Y. Gogotsi, Nat. Mater., 2008, 7, 845-854.

13 J. R. Miller and P. Simon, Science, 2008, 321, 651-653.

14 C. Arbizzani, M. Mastragostino and F. Soavi, J. Power Sources, 2001, 100, 164-170.

15 A. Burke, Electrochim. Acta, 2007, 53, 1083-1091.

16 R. Kötz and M. Carlen, Electrochim. Acta, 2000, 45, 24832498.

17 Y. T. Li, Y. T. Pi, L. M. Lu, S. H. Xu and T. Z. Ren, J. Power Sources, 2015, 299, 519-528.

18 B. D. Hulicova-jurcakova, M. Seredych, G. Q. Lu and J. Bandosz, Adv. Funct. Mater., 2009, 19, 438-447.

19 X. Ying, C. Chen, Z. Jie, D. Hua, X. Deng and J. Wei, J. Power Sources, 2013, 230, 50-58.

20 M. Inagaki, H. Konno and O. Tanaike, J. Power Sources, 2010, 195, 7880-7903.

21 A. Chen, Y. Wang, Y. Yu, H. Sun, Y. Li, K. Xia and S. Li, J. Mater. Sci., 2017, 52, 3153-3161.

22 B. Duan, X. Gao, X. Yao, Y. Fang, L. Huang, J. Zhou and L. Zhang, Nano Energy, 2016, 27, 482-491.
23 J. Chang, Z. Gao, X. Wang, D. Wu, F. Xu, X. Wang, Y. Guo and K. Jiang, Electrochim. Acta, 2015, 157, 290-298.

24 J. P. Olivier, Carbon, 1998, 36, 1469-1472.

25 M. Thommes, K. Kaneko, A. V. Neimark, J. P. Olivier, F. Rodriguez-Reinoso, J. Rouquerol and K. S. W. Sing, Pure Appl. Chem., 2015, 87, 1051-1069.

26 M. S. Dresselhaus, G. Dresselhaus, R. Saito and A. Jorio, Phys. Rep., 2005, 409, 47-99.

27 M. S. Dresselhaus, A. Jorio, M. Hofmann, G. Dresselhaus and R. Saito, Nano Lett., 2010, 10, 751-758.

28 T. C. Chieu, M. S. Dresselhaus and M. Endo, Phys. Rev. B: Condens. Matter Mater. Phys., 1982, 26, 5867-5877.

29 C. $\mathrm{Hu}, \mathrm{S}$. Sedghi, A. Silvestre-Albero, G. G. Andersson, A. Sharma, P. Pendleton, F. Rodrguez-Reinoso, K. Kaneko and M. J. Biggs, Carbon, 2015, 85, 147-158.

30 H. Wang, Z. Xu, A. Kohandehghan, Z. Li, K. Cui, X. Tan, T. J. Stephenson, C. K. King, C. M. B. Holt, B. C. Olsen, J. K. Tak, D. Harfield, A. O. Anyia and D. Mitlin, ACS Nano, 2013, 7, 5131-5141.

31 R. Arrigo, M. Ha, S. Wrabetz, R. Blume, M. Lerch, J. Mcgregor, E. P. J. Parrott, J. A. Zeitler, L. F. Gladden, A. Knop-gericke, R. Schlo and D. S. Su, J. Am. Chem. Soc., 2010, 132, 9616-9630.

32 M. Zhou, F. Pu, Z. Wang and S. Guan, Carbon, 2013, 68, 185194.

33 M. Yang and Z. Zhou, Adv. Sci., 2017, 1600408.

34 N. Guo, M. Li, Y. Wang, X. Sun, F. Wang and R. Yang, RSC Adv., 2016, 6, 101372-101379.

35 J. R. Pels, F. Kapteijn, J. A. Moulijn, Q. Zhu and K. M. Thomas, Carbon, 1995, 33, 1641-1653.

36 J. Casanovas, J. M. Ricart, J. Rubio, F. Illas and J. M. JiménezMateos, J. Am. Chem. Soc., 1996, 118, 8071-8076.

37 C. T. Hsieh and H. Teng, Carbon, 2002, 40, 667-674.

38 C. Chen, D. Yu, G. Zhao, B. Du, W. Tang, L. Sun, Y. Sun, F. Besenbacher and M. Yu, Nano Energy, 2016, 27, 377-389.

39 Y. Zhao, M. Lu, P. Tao, Y. Zhang, X. Gong, Z. Yang, G. Zhang and H. Li, J. Power Sources, 2016, 307, 391-400. 